\title{
GW23-e1315 ASSESSMENT OF LEFT VENTRICULAR GLOBAL SYSTOLIC FUNCTION IN LONG-TERM SURVIVAL PATIENTS OVER HT OPERATION BY SPECKLE TRACKING IMAGING
}

doi:10.1136/heartjnl-2012-302920ad.45

Xiaojuan Qin, Mingxing Xie. Department of Ultrasonography, Union Hospital of Tongji Medical College, Huazhong University of Science and Technology, Hubei Provincial Key Laboratory of Molecular Imaging, Wuhan 430022, China

Objectives Left ventricular hypertrophy (LVH) caused myocardial dysfunction. LVH in postoperative patients with orthotopic heart transplantation was caused by variety factors. The aim of the study was to analysis the influence of LVH on left ventricular global systolic function by speckle tracking imaging (STI).

Methods 24 patients, who live longer than 1 year after heart transplantation, and 30 healthy volunteers are examined with twodimensional echocardiography and Colour Doppler Flow Imaging (CDFI). These 24 patients are divided into two groups: one group of patients with left ventricular hypertrophy (HT-LVH), another with normal left ventricular mass (HT-NLVM). The short axis view of the left ventricular papillary muscle and the apical fourchamber view are acquired and stored in dynamic mode. Then they are analysed off-line in Olab Analysis 7.1. We compare the global left ventricular longitudinal strain (GLS), global circumferential strain (GCS), global radial strain (GRS), GLS rate (GLSR), GCS strain (GCSR) and GRS strain (GRSR). The global left ventricular function is evaluated, and the possible reasons are analysed.

\section{Results}

1. Left ventricular ejection fraction (LVEF) was no significant differences among the normal control group, HT-NLVM, HT-LVH $(64.00 \pm 4.49$ vs $62.65 \pm 4.73$ vs $65.21 \pm 3.71 \mathrm{~cm}$, all $p>0.05)$. But IVST, PWT, LVM of three group, these of HT-LVH was largest, and HT-NLVM was larger (all $\mathrm{p}<0.05$ ). LVEDD was no significant difference between normal control and HT-NLVM, but LVEDD of HT-LVH was higher than that of control and HTNLVM (all $\mathrm{p}<0.05)$.

2. GRS, GRSR, GCS, GCSR, GLS, GLSR of HT-NLVM were lower than those of normal control $(20.95 \pm 4.34$ vs $29.69 \pm 3.38,-2.26$ \pm 0.70 vs $-3.02 \pm 0.49,-16.95 \pm 2.98$ vs $-19.17 \pm 2.00,1.48 \pm 0.35$ vs $1.72 \pm 0.28,-16.00 \pm 2.52$ vs $-17.96 \pm 1.63,1.39 \pm 0.33$ vs 1.64 \pm 0.25 , respectively; all $p<0.05$ ), and those of HT-LVH were further lower than those of HT-NLVM $(19.87 \pm 3.85$ vs 20.95 $\pm 4.34,-1.85 \pm 0.78$ vs $-2.26 \pm 0.70,-14.19 \pm 2.17$ vs -16.95 $\pm 2.98,1.22 \pm 0.21$ vs $1.48 \pm 0.35,-13.10 \pm 1.25$ vs $-16.00 \pm 2.52$, $1.12 \pm 0.18$ vs $1.39 \pm 0.33$, respectively; all $p<0.05$ ).

3. GRS, GRSR, GCS, GCSR, GLS, GLSR were affected by increasing of LVM. The predictive value $\mathrm{R}^{2}$ of LVM for GRS, GRSR, GCS, GCSR, GLS, GLSR was 0.327, 0.224, 0.393, 0.369, 0.325, 


\section{ABSTRACTS}

0.231 , respectively (all $\mathrm{p}<0.01$ ).

Conclusions Increasing of LVM in postoperative patients with HT resulted in left ventricular global systolic dysfunction. The prevention of postoperative LVH is beneficial to maintenance of LV myocardium contraction function. 